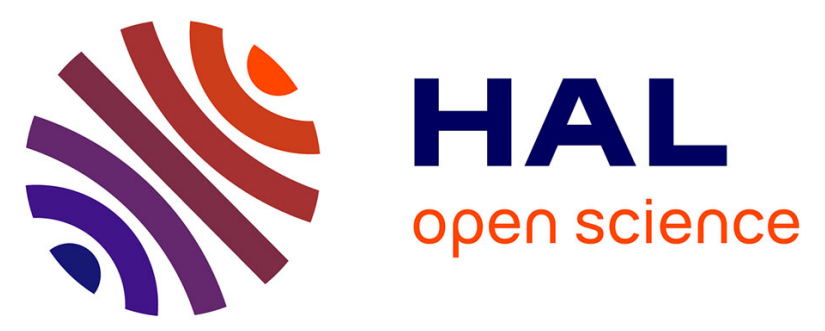

\title{
Native Collagen: Electrospinning of Pure, Cross-Linker-Free, Self-Supported Membrane
}

Dounia Dems, Julien Rodrigues da Silva, Christophe Hélary, Frank Wien, Marion Marchand, Nicolas Debons, Laurent Muller, Yong Chen, Marie-Claire Schanne-Klein, Christel Laberty-Robert, et al.

\section{To cite this version:}

Dounia Dems, Julien Rodrigues da Silva, Christophe Hélary, Frank Wien, Marion Marchand, et al.. Native Collagen: Electrospinning of Pure, Cross-Linker-Free, Self-Supported Membrane. ACS Applied Bio Materials, 2020, 10.1021/acsabm.0c00006 . hal-02541707

\section{HAL Id: hal-02541707 https://hal.science/hal-02541707}

Submitted on 14 Apr 2020

HAL is a multi-disciplinary open access archive for the deposit and dissemination of scientific research documents, whether they are published or not. The documents may come from teaching and research institutions in France or abroad, or from public or private research centers.
L'archive ouverte pluridisciplinaire HAL, est destinée au dépôt et à la diffusion de documents scientifiques de niveau recherche, publiés ou non, émanant des établissements d'enseignement et de recherche français ou étrangers, des laboratoires publics ou privés. 


\section{Native collagen: electrospinning of pure, cross- linker free self-supported membrane}

Dounia Dems, ${ }^{a}$ Julien Rodrigues da Silva,${ }^{a}$ Christophe Hélary, ${ }^{a}$ Frank Wien, ${ }^{b}$ Marion Marchand, ${ }^{c}$ Nicolas Debons, ${ }^{a}$ Laurent Muller, ${ }^{c}$ Yong Chen, ${ }^{d}$ Marie-Claire Schanne-Klein, ${ }^{e}$ Christel Laberty-Robert, ${ }^{a}$ Natacha Krins, ${ }^{a}$ Carole Aimé ${ }^{a},{ }^{*}$

a. Sorbonne Université, CNRS, Laboratoire de Chimie de la Matière Condensée de Paris (LCMCP), Paris, F-75005, France

b. SOLEIL Synchrotron, Saint Aubin 91190, France

c. Center for Interdisciplinary Research in Biology (CIRB), Collège de France, CNRS, INSERM, PSL Research University, Paris, France

d. Ecole Normale Supérieure, CNRS-ENS-SU UMR 8640, 24 rue Lhomond, Paris, 75005, France

e. Laboratoire d'Optique et Biosciences, Ecole Polytechnique, CNRS, Inserm, Institut Polytechnique de Paris, F-91128 Palaiseau, France

ABSTRACT. Rebuilding biological environments is crucial when facing the challenges of fundamental and biomedical research. Thus, preserving the native state of biomolecules is essential. We use electrospinning (ES), which is an extremely promising method for the preparation of fibrillar membranes to mimic the ECM of native tissues. Here, we report for the 
first time (1) the ES of pure and native collagen into a self-supported membrane in absence of crosslinker and polymer support, (2) the preservation of the membrane integrity in hydrated media in absence of crosslinker and (3) the preservation of the native molecular structure and recovery of the hierarchical assembly of collagen. We use a multiscale approach to characterize collagen native structure at the molecular level using circular dichroism, and to investigate collagen hierarchical organization within the self-supported membrane using a combination of multiphoton and electron microscopies. Finally, we show that the membranes are perfectly suited for cell adhesion and spreading, making them very promising candidates for the development of biomaterials and finding applications in biomedical research.

KEYWORDS. Collagen; electrospinning; hierarchical organization; extra-cellular matrix; tissue engineering; second harmonic generation microscopy

INTRODUCTION. Increasing the biological relevance of in vitro models is an ever-increasing goal in biological and medical research. This implies preserving the native state of living systems, i.e. their native conformation, multi-scale organization, molecular recognition properties. Mimicking the structure of the extracellular matrix (ECM) is of primary importance to provide the adequate tridimensional (3D) environment and biological cues for cell attachment, proliferation and function. This key 3D organization mainly arises from the hierarchical structure of type I collagen, the major component of ECM. Collagen triple helices self-assemble into fibrils of 20 to $500 \mathrm{~nm}$ in diameter. It is therefore crucial to preserve its native state when processing biologically relevant scaffolds. Electrospinning (ES) has become increasingly popular as a cost effective technology to produce nanofiber scaffolds for tissue engineering, providing self-supported membranes with controlled porosity. ${ }^{1,2}$ ES allows to produce fibers with tunable diameter (smaller 
than $1 \mu \mathrm{m}$ ) and orientation. Biopolymers are the most relevant candidates to be electrospun. However, ES of collagen remains challenging. Collagen solutions do not have the viscoelastic response necessary for jet stabilization partly due to the stability of collagen tertiary structure..$^{3,4}$ As a result, denaturing fluoroalcohols are often used, such as 1,1,1,3,3,3-hexafluoro-2-propanol ${ }^{5-}$ ${ }^{10}$ and 2,2,2-trifluoroethanol, ${ }^{8}$ allowing stretching and rearrangement of collagen. Since then, the preservation of the native state of collagen has continuously been a subject of debate. ${ }^{11,12}$ Several works evidence that collagen completely unfolds in fluorinated solvents. Efforts have been made to find alternative solvents, such as phosphate buffer saline (PBS)/ethanol ${ }^{13}$ or acetic acid/DMSO ${ }^{14}$ mixtures. All of them involve the use of chemical crosslinkers (glutaraldehyde ${ }^{5,15}$ or carbodiimide $\mathrm{e}^{13,14,16,17,18}$ ) to prevent the membrane from falling apart in hydrated environment. However, the use of crosslinkers alters the fibrillogenesis of collagen and the biocompatibility of the materials. ${ }^{19}$ Original approaches have been developed using a polymer support, where collagen is electrospun together with a synthetic polymer (polycaprolactone, ${ }^{7,8,20,21}$ polyethylene oxide, $^{18,22,23}$ polyvinyl-pyrrolidone ${ }^{24}$ ) eventually washed out after crosslinking, or with a biopolymer ${ }^{25,26}$ including elastin, ${ }^{18}$ chitosan, ${ }^{9}$ or gelatin. ${ }^{27}$

In this work, we report for the first time the ES of pure collagen into a self-supported membrane in absence of polymer support (Fig. 1A). The key is to use an aqueous acidic/alcoholic solvent mixture. We also stabilize the membrane structure for further applications in biological hydrated environment in absence of crosslinkers by inducing collagen pre-fibrillation in basic conditions. Since the hierarchical structure of collagen is crucial, we present a multiscale characterization approach to probe the material from its molecular- up to its micro-scale. We claim this approach to be a key step for collagen-based material characterization. It combines highly specific and biologically adapted methods including multiphoton microscopy based on second 
harmonic generation (SHG), field emission scanning electron microscopy in a cryo mode (CryoFE-SEM) and synchrotron radiation-circular dichroism (SRCD).

Mastering native collagen electrospun membranes is the base for building more advanced biomaterials and use them as a support to study even further the environment of living cells. As an example we show the processing of hybrid biomaterials and the use of pure collagen membrane into microfabricated chips for biological microfluidics.

A

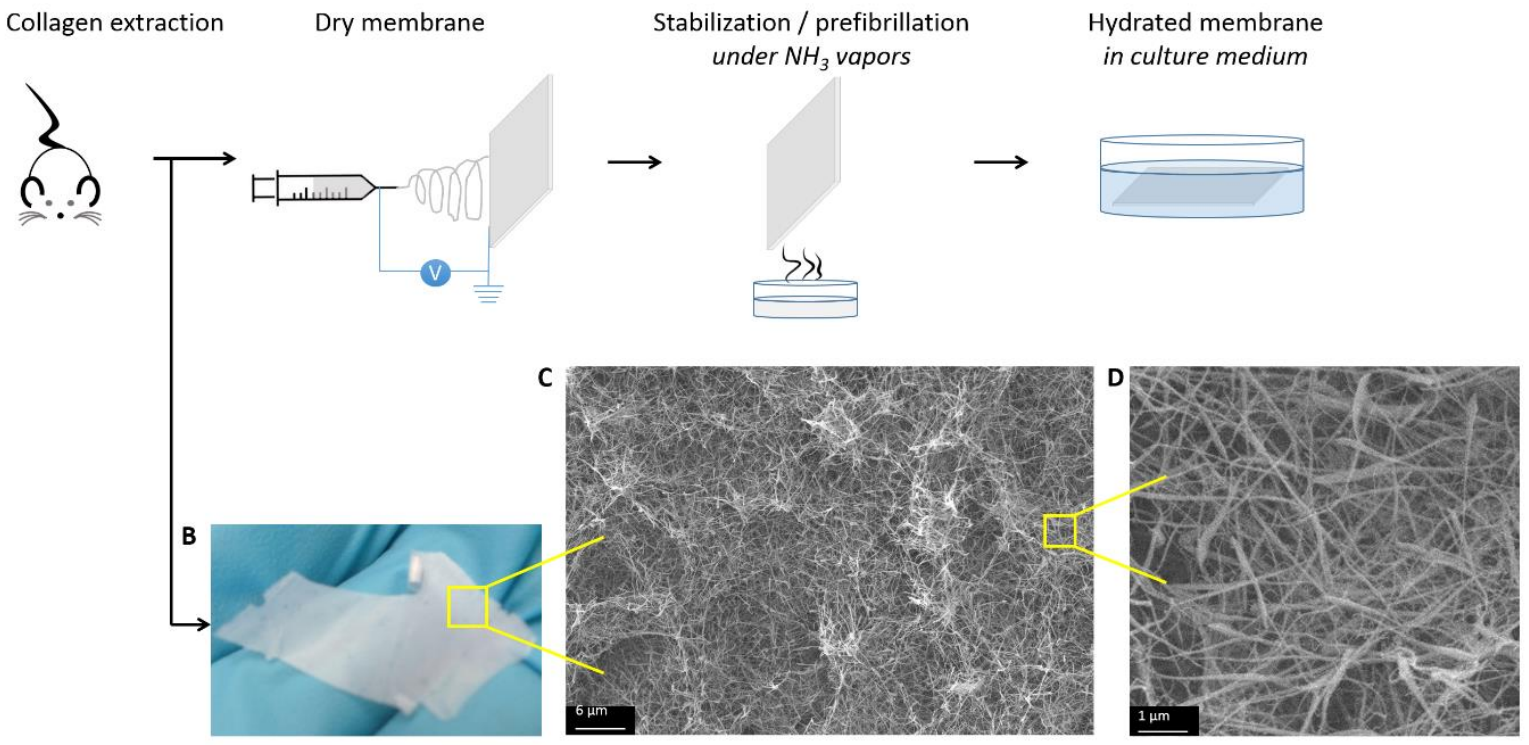

Figure 1. (A) Scheme of collagen ES and membrane processing. (B) Photo, (C,D) SEM images of a self-supported membrane ([collagen] $=1.7 \mathrm{mg} \cdot \mathrm{mL}^{-1}$ in $30 \mathrm{mM} \mathrm{HCl}$ :EtOH $\left.(25: 75 \mathrm{v} / \mathrm{v})\right)$.

\section{EXPERIMENTAL SECTION.}

Collagen extraction and purification. Type I collagen was extracted and purified from rat tail tendons as previously described by substituting $500 \mathrm{mM}$ acetic acid with $3 \mathrm{mM}$ hydrochloric acid. ${ }^{28,29}$ Collagen purity was assessed by electrophoresis and its concentration estimated by 
hydroxyproline titration. ${ }^{30}$ All other chemicals were purchased and used as received. Water was purified with a Direct-Q system (Millipore Co.).

Collagen electrospinning. $1.2 \mathrm{~mL}$ of the solution was electrospun for 4 hours at ca. $12 \mathrm{kV}$ with a flow rate of $0.005 \mathrm{~mL} \cdot \mathrm{min}^{-1}$ in a home-made chamber flushed with dry air (ca. 10\% humidity) at room temperature $\left(\sim 20^{\circ} \mathrm{C}\right)$. Humidity is a key parameter to be controlled to ensure the good electrospinning of collagen and the reproducibility of the experiment. For this reason, we used synthetic dry air (Air Liquid). The counter electrode was a copper tape $(2.5 * 1.5 \mathrm{~cm})$ fixed on a Teflon support. The distance between the metal needle and the counter electrode was $9 \mathrm{~cm}$. After processing, the electrospun membrane was detached from the copper support, and a homogeneous self-standing membrane was obtained. Casted collagen membranes were prepared as control and obtained by pouring $1.2 \mathrm{~mL}$ of a collagen solution at a concentration of $1.7 \mathrm{mg}^{-\mathrm{ml}^{-1}}$ in $30 \mathrm{mM} \mathrm{HCl}$ and $75 \%$ vol. ethanol into a Teflon mold. The solvent was allowed to evaporate overnight under ambient conditions.

Pre-fibrillation of electrospun membranes by ammonia exposure. $4 \mathrm{~mL}$ of ammonium hydroxide solution (25\%, Carlo Erba) in a glass beaker were placed in a desiccator $(153 * 191 \mathrm{~mm})$. After 20 min exposure to ammonia vapors, collagen membranes were rinsed three times a day for 10 days in culture medium to remove all ammonia residues before cell proliferation testing.

Synchrotron-radiation circular dichroism. $25 \mu \mathrm{L}$ of collagen solutions in $3 \mathrm{mM} \mathrm{HCl}$ were loaded in quartz cells (Hellma) with a path length of $0.01 \mathrm{~cm}$. The measurements were performed on the DISCO Beamline at SOLEIL synchrotron (Saint Aubin, France). ${ }^{31,32}$ Melting temperatures were obtained by increasing temperature from 20 to $74^{\circ} \mathrm{C}$ with steps of $3{ }^{\circ} \mathrm{C}$ and an equilibration time of $5 \mathrm{~min}$. Raw spectra were acquired from 320 to $170 \mathrm{~nm}$ with a $1 \mathrm{~nm}$ spectral resolution. 
Spectra were treated with the CDTool software. ${ }^{33}$ They are the average of three spectra. A background ( $\mathrm{HCl} 3 \mathrm{mM}$ acquired in the same conditions) was subtracted from them. Intensity calibration was obtained with a CSA sample.

Second harmonic generation / 2-photon excited fluorescence. We used a custom-built laserscanning multiphoton microscope and recorded Second Harmonic Generation (SHG) and 2-photon excited fluorescence (2PEF) images in parallel as previously described. ${ }^{34}$ Excitation was provided by a femtosecond titanium-sapphire laser (Mai-Tai, Spectra-Physics) tuned to $860 \mathrm{~nm}$, scanned in the $\mathrm{XY}$ directions using galvanometric mirrors and focused using a $25 \times$ objective lens (XLPLN25XWMP2, Olympus), with a resolution of typically $0.4 \mu \mathrm{m}$ (lateral) $\times 1.7 \mu \mathrm{m}$ (axial). We used either circular polarization in order to image all structures independently of their orientation in the image plane, or a set of linear polarizations with different orientations in order to perform polarization-resolved measurements. All polarization-resolved images were acquired at 36 excitation angles $\theta$ regularly spaced between $0^{\circ}$ and $360^{\circ}$, using $200 \mathrm{kHz}$ acquisition rate and $300 \times 300 \mathrm{~nm}^{2}$ pixel size. Power at the sample was typically $12 \mathrm{~mW}$ for dry and stabilized membranes, and $2 \mathrm{~mW}$ for the hydrated samples. Epi-detection was used for dry and stabilized membranes and $2 \times 2$ binning was applied before pSHG processing. Transdetection was used for hydrated sample, with no binning. pSHG images were processed as already described ${ }^{34}$ to provide two images: (i) the average of all images acquired with the set of linear polarizations, that is similar to an SHG image acquired with circular polarization; (ii) a map of the main orientation of collagen in the image plane. The latter pSHG image is displayed using the HSV Look-up-table, where $\mathrm{H}$ is the orientation displayed in the insert, and $\mathrm{V}$ is the brightness, which is set to 1 if the pSHG processing is satisfactory $(\mathrm{R} 2>0.5)$. Three areas were observed on each sample to check for the sample structure homogeneity. Each sample was investigated in triplicate. 
SEM observations. SEM imaging was performed using a Variable Pressure Hitachi S-3400N working at an accelerating voltage of $10 \mathrm{kV}$, using an in lens secondary electron (SE) detector, with a working distance at / around $4.2 \mathrm{~mm}$. Self-supported membranes were deposited on carbontape coated aluminum pads. Samples were coated with a $10 \mathrm{~nm}$ gold layer before observations.

Cryo-FE-SEM observations. Sample preparation and observations were performed at the Electron Microscopy Facility (EMF) of the Institut de Biologie Paris-Seine (Sorbonne University, Paris). Prior to observations, samples were sandwiched between two cupules then cryo fixed by immersion in liquid ethane using a cryofixation station (CPC, Leica). Freeze-fracture was processed at $-150^{\circ} \mathrm{C}$ using a high vacuum station (ACE 600, Leica). FE-SEM observations were performed at $-120^{\circ} \mathrm{C}$ at low voltage $(0.790 \mathrm{kV})$ using an in lens secondary electron (SE) detector, with a $20 \mu \mathrm{m}$ objective aperture diameter and a working distance at / around $2.5 \mathrm{~mm}$ (GeminiSEM 500, Zeiss; Cryo station VCT100, Leica). A sublimation step at $-90^{\circ} \mathrm{C}$ was applied for $15 \mathrm{~min}$ in the SEM chamber to remove ice. Scan speed and line or drift compensation integrations were adjusted during observations.

Cell culture experiments. Normal Human Dermal Fibroblasts (NHDF) were cultured in complete cell culture medium: Dulbecco's Modified Eagle's Medium (DMEM) supplemented with 10\% fetal serum, $100 \mathrm{U} \cdot \mathrm{mL}^{-1}$ penicillin, $100 \mu \mathrm{g} \cdot \mathrm{mL}^{-1}$ streptomycin, $0.25 \mu \mathrm{g} \cdot \mathrm{mL}^{-1}$ Fungizone, and glutamax. Tissue culture flasks $\left(75 \mathrm{~cm}^{2}\right)$ were kept at $37^{\circ} \mathrm{C}$ in a $95 \%$ air : $5 \% \mathrm{CO}_{2}$ atmosphere. Before confluence, cells were removed from culture flasks by treatment with $0.1 \%$ trypsin and $0.02 \%$ EDTA. Cells were rinsed and suspended in complete culture medium before use.

After pre-fibrillation of the collagen membranes (either electrospun or casted, see below section S3), NHDF were cultured for 24 hours on the surface of membranes at a cell seeding density of 2 
kCells $/ \mathrm{mm}^{2}$. Cells were incubated at $37^{\circ} \mathrm{C}$ in a $95 \%$ air : $5 \% \mathrm{CO}_{2}$ atmosphere. Cell culture experiments were performed three times in triplicate $(\mathrm{n}=9)$.

Long term cytotoxicity of collagen membranes on NHDF. Collagen membranes (casted, $n=3$, or electrospun, $n=3$ ) were set into a 48 well plate. Then, 100,000 NHDF were seeded per well. Cells were incubated for 7 days in contact with membranes in $200 \mu \mathrm{L}$ of colorless DMEM complete medium at $37^{\circ} \mathrm{C}$ in a $95 \%$ air $-5 \% \mathrm{CO}_{2}$ atmosphere. Cell metabolic activity was monitored at day 7 using Alamar Blue assay. For this purpose, fibroblasts were incubated with 300 $\mu \mathrm{L}$ of a rezazurin solution at $0.1 \%(\mathrm{w} / \mathrm{v})$ for 4 hours. The supernatant in each well was then collected, diluted with $700 \mu \mathrm{L}$ of fresh colorless medium and the absorbance measured at $\lambda=570$ $\mathrm{nm}$ and $\lambda=600 \mathrm{~nm}$. The percentage of rezazurin reduction was calculated following the formula provided by the supplier. Cell metabolic activity of each well was compared to control samples, i.e. cells cultivated without membranes. The arbitrary value $100 \%$ was given to control samples.

Fluorescence microscopy (evaluation of cytocompatibility). Collagen membranes were fixed with 4\% paraformaldehyde in PBS for 1 hour, rinsed three times with PBS for 5 min each and incubated with PBS $0.2 \%$ Triton for 20 min. They were then incubated with Alexa Fluor 488 Phalloidin (Molecular Probe ${ }^{\circledR}$ ) diluted 1/200 (v/v) in PBS in a dark chamber for 45 min. After rinsing three times for $5 \mathrm{~min}$ each in PBS, the samples were incubated with DAPI (Molecular Probe ${ }^{\circledR}$ ) diluted $1 / 50,000$ in PBS in a dark chamber for 10 min. Finally, the membranes were washed three times with PBS and mounted between slides and coverslips with the antifading solution AF3 (Biovalley) for fluorescence microscopy (ZEISS Axioplan microscope). Fibroblasts were observed at magnification $\times 100$ and $\times 400$.

\section{RESULTS AND DISCUSSION.}




\section{A self-supported membrane}

Electrospinning is particularly relevant for the design of 3D fibrillary scaffold. In the ES process, high voltage is applied to a polymer solution that is ejected through a needle towards a grounded collector as dried fibers. Upon voltage application, the liquid droplet deforms into a cone, the "Taylor cone", and emits a charged jet of liquid. Because of the low charge of collagen, obtaining a self-supported membrane is not an easy task. It requires adjusting many parameters to get a stable jet and ensure nanofiber formation (Fig. S1 and S2). ${ }^{35}$ Solvent is an important factor for biopolymer stability and stretching during ES and for the drying of the mesh on the collector. Collagen was dissolved in a mixture of hydrochloric acid $(3 \mathrm{mM} \mathrm{HCl}, \mathrm{pH} 2.5)$ and ethanol (75\% vol) with a final concentration of $1.7 \mathrm{mg} \cdot \mathrm{mL}^{-1}$. In those carefully adjusted conditions, a selfsupported membrane was obtained with a thickness of $40 \mu \mathrm{m}$ exhibiting randomly oriented and interconnected fibrils with diameter in the nanometer range $(58 \pm 10 \mathrm{~nm})$ (Fig. $1 \mathrm{~B}-\mathrm{D}$, and Fig. S2).

The stabilization of the membrane structure in a hydrated form is crucial for biomedical applications. When immersing the electrospun collagen in cell culture medium, the membrane falls apart. To tackle this issue, works reported in the literature often use chemical cross-linkers that can be detrimental for cell survival, while hampering collagen hierarchical self-assembly. Alternative strategies have to be developed to strengthen the hierarchical structure of collagen. To this aim, we exposed ES membranes to ammonia vapors, to take advantage of the increase in $\mathrm{pH}$ to stabilize collagen fibrils. This strategy is known to trigger fibrillogenesis and gelling in collagen solution ${ }^{36-}$

${ }^{38}$ but is here adapted to dry materials. After a 20 minutes exposure of the dry membrane, the macroscopic structure of membranes was unchanged. The stabilized biomaterials could be stored 
in culture medium over months (Fig. S3-S5) with mechanical properties characterized by a mean Young's modulus of ca $6 \mathrm{kPa}$ (Fig. S6 and Supplementary experimental section).

Whether collagen may be denaturated during ES remains an open question in literature. This can involve both the solvent used for improving the electrospinnability of the collagen or the spinning process by itself and the high voltage applied. This question is addressed in the following sections through a multiscale characterization from the micron to the molecular scale.

\section{Hierarchical organization of collagen}

In living tissues, collagen self-assembles into fibrils, with triple helices all oriented in the same direction with the same polarity. SHG is a coherent process specific for non- centrosymmetric materials. At the molecular level, it relies on nonlinear dipole excitation along the peptide bonds. It builds up efficiently in highly anisotropic fibrillar collagen because of the tight unidirectional alignment (parallel) of peptide bonds along the collagen triple helix and within fibrils both in isolated collagen fibrils ${ }^{39,40}$ and fibrillary networks. ${ }^{41}$ This makes SHG microscopy the gold standard technique for 3D characterization of collagen-rich tissues in ambient conditions and without exogeneous labelling, with a structural specificity that cannot be reached with any other optical and fluorescence microscope, being specific to (i) the native state of collagen (over gelatin) and (ii) the unidirectional alignment of collagen triple helices. ${ }^{42}$ 
A

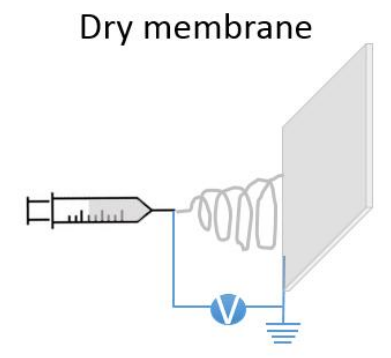

A1

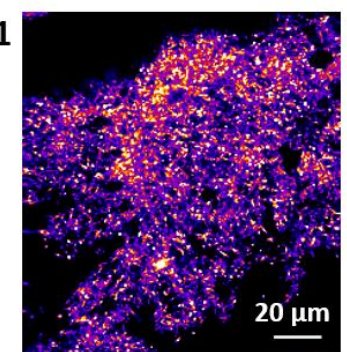

A2

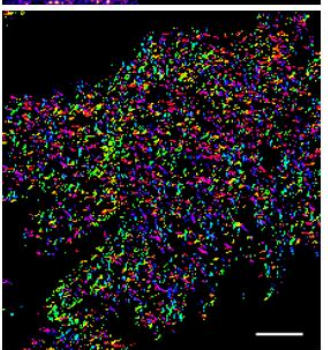

A3

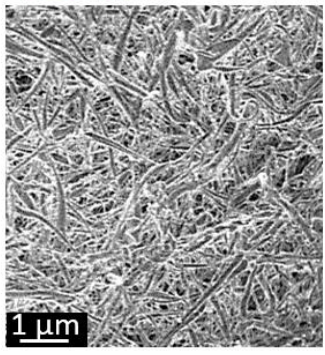

B

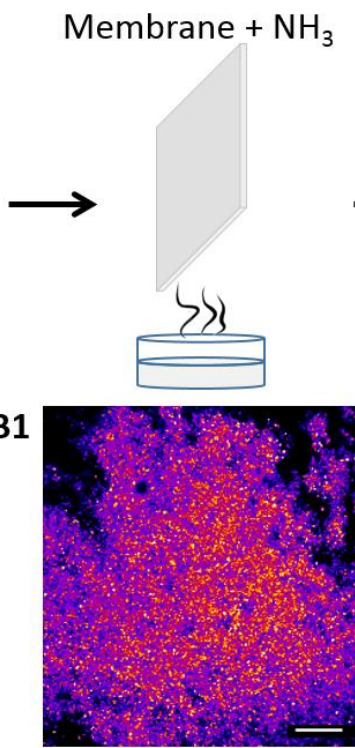

B2

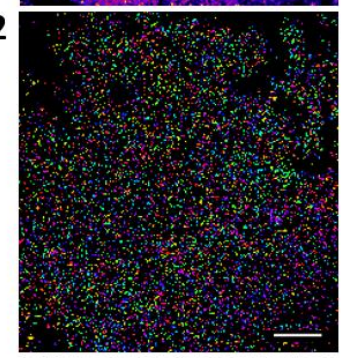

B3

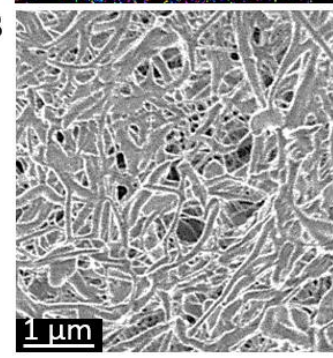

C

Hydrated membrane
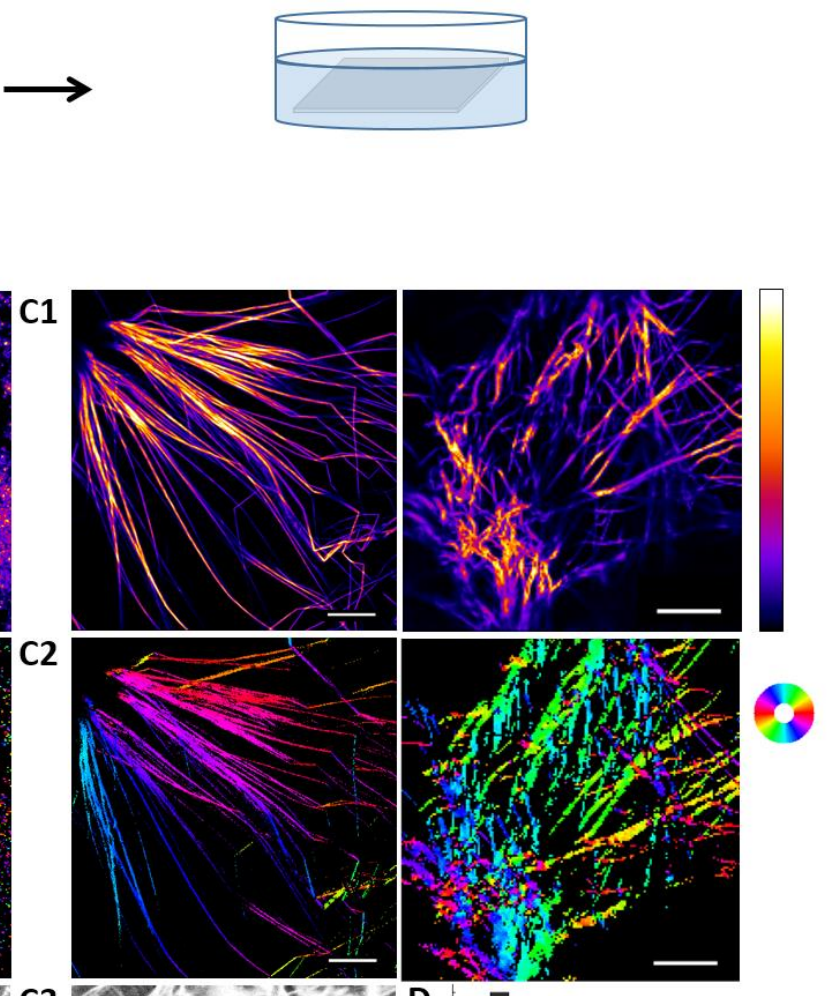

C3
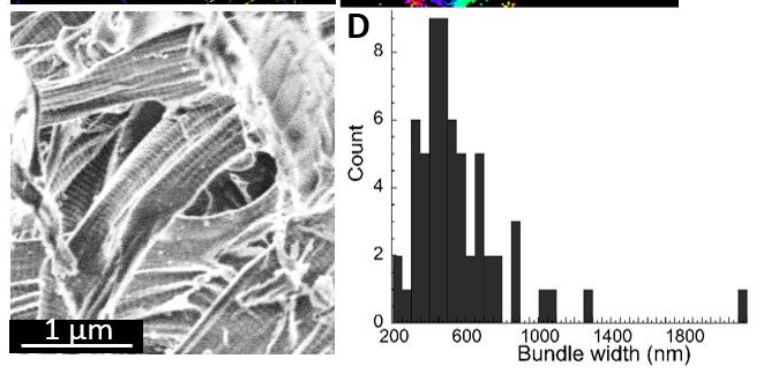

Figure 2. (1) SHG, (2) pSHG and (3) cryo-FE-SEM images of (A) a dry collagen membrane, (B) after exposure to ammonia vapors and $(\mathrm{C})$ rehydration in culture medium. The look-up table (on the right in the A1-C1 line) corresponds to arbitrary units for each SHG image. The orientation in the pSHG image is displayed on the colored circular scale (on the right in the A2-C2 line) $\left(\left[\right.\right.$ collagen] $=1.7 \mathrm{mg} \cdot \mathrm{mL}^{-1}$ in $\left.30 \mathrm{mM} \mathrm{HCl}: \mathrm{EtOH}(25: 75 \mathrm{v} / \mathrm{v})\right)$. Scale bar for SHG and pSHG is 20 $\mu \mathrm{m}$. (D) Histogram of bundle width distribution according to cryo-FE-SEM. 
Measurements on dry membranes showed a high SHG signal (Fig. 2A1). This is a proof of the native state of collagen that corresponds to a non-centrosymmetric packing of the triple helices. In addition, the grainy-like pattern with a myriad of dots indicates the presence of small structures that are highly entangled, in agreement with SEM observations (Fig. 1C,D). Indeed, given that the diameter of the fibrils (58 $\pm 10 \mathrm{~nm}$ according to SEM images) is small relatively to the focal volume (typically $0.4 \mu \mathrm{m}$ (lateral) $\times 1.7 \mu \mathrm{m}$ (axial)), many fibrils may be simultaneously observed in one pixel. At the intersection of two fibrils, molecules in the focal volume are not all oriented in the same direction, which results in a centrosymmetric distribution and leads to a local decrease of SHG signal (Fig. 3A1). If the network is highly entangled, SHG decrease is widespread throughout the focal volume leading to a grainy-like image (Fig. 3A2-A3). The structural specificity of SHG images has been further improved by acquiring polarization-resolved images (pSHG), which measures the main orientation of collagen in the imaging plane, in every pixel of the SHG image. ${ }^{34}$ Given that each color codes for a given direction, the presence of dots with many different colors throughout the whole image shows that the highly packed structures of native collagen are randomly distributed with respect to one another (Fig. 2A2). Again, at fibrils intersection, all molecules are not oriented in the same direction, which impedes the determination of a main orientation, resulting in black pixels (Fig. 3B). 

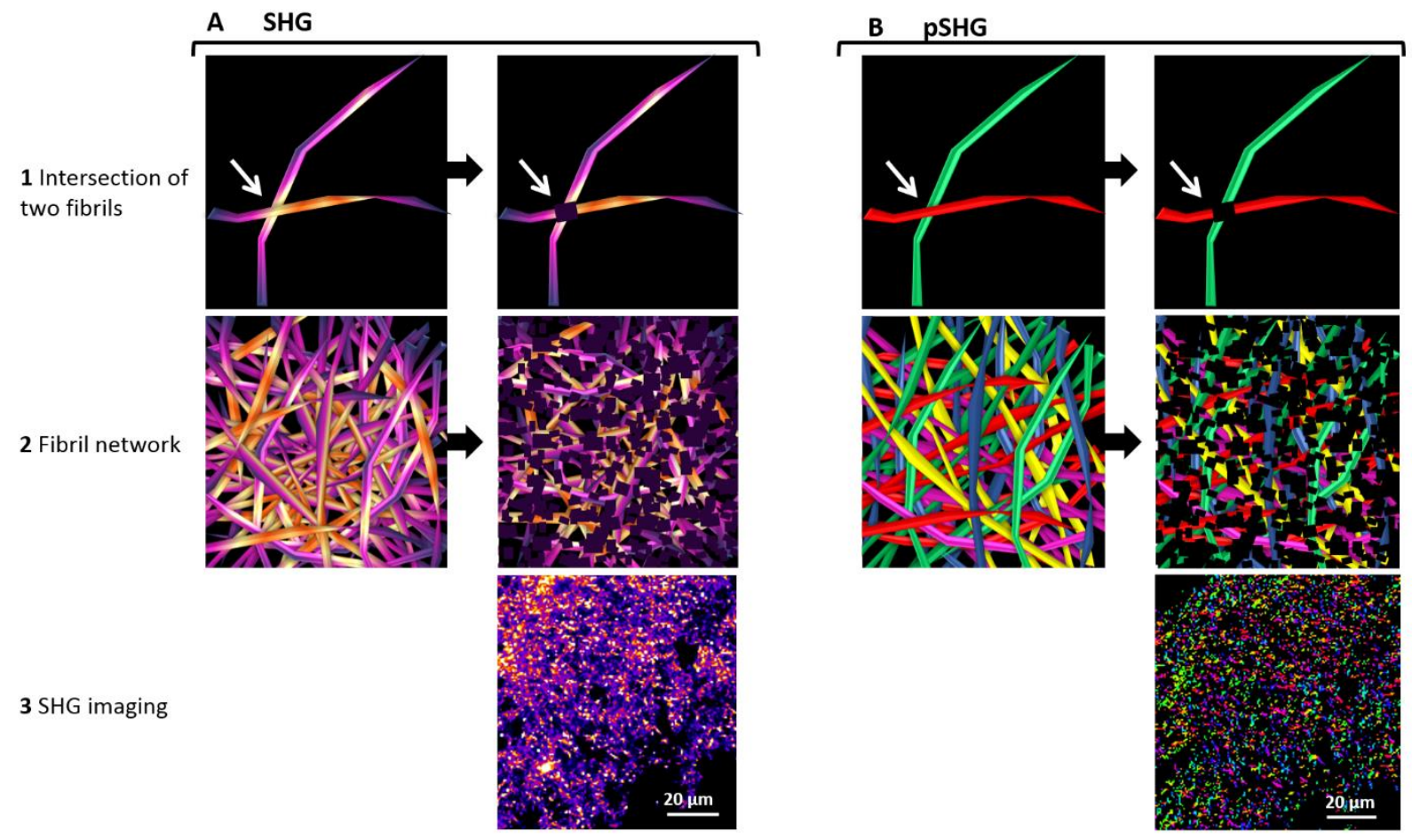

Figure 3. Schematic representation of the local decrease in (A) SHG and (B) pSHG intensity: (1) at the intersection of two fibrils, and (2) in highly entangled network of small diameter-fibers. (3) Corresponding SHG images with a grainy-like pattern ([collagen] $=1.7 \mathrm{mg} \cdot \mathrm{mL}^{-1}$ in $30 \mathrm{mM}$ $\mathrm{HCl}: \mathrm{EtOH}(25: 75 \mathrm{v} / \mathrm{v}))$.

After 20 min exposure to $\mathrm{NH}_{3}$ vapors, no variation of the SHG and pSHG profiles could be detected (Fig. 2B1-2). This shows the preservation of the triple helix structure and packing within small entangled structures. No important variation at the scale probed by SHG can be detected despite the very different behaviors of the dry and stabilized membranes when immersed in culture medium.

In contrary, SHG observations of the hydrated membrane showed very important variations of the membrane structure that appears made up of large micrometer-long fibrils forming ca. $100 \mu \mathrm{m}-$ 
large domains (Fig. 2C1). This is reminiscent of collagen matrices in dense fibrillated media. pSHG very nicely reveals the unidirectional ordering of the triple helices in each collagen fibril that appears as monocolored structures (Fig. 2C2). In this case, the absence of grainy-like profile is attributed to the fact that collagen reorganization leads to the formation of large structures. Indeed, one of the major features of type I collagen is its ability to self-assemble into highly organized supramolecular structures, which in turn form larger highly oriented fibers and fascicles. This prevents the observation of multiple collagen fibrils with random organization with respect to each other within the focal volume, hence precluding local SHG extinction.

Further characterization of collagen triple helix arrangement can be obtained at the nanometer scale by use of electron microscopy. We used cryo-FE-SEM that preserves the hydrated state of collagen in hydrated biomaterials. The dry membrane exhibits the same structure as observed under conventional SEM (Fig. 1) due to the absence of solvent within the initial biomaterial: randomly entangled fibrils are clearly visible (Fig. 2A3). Once stabilized under $\mathrm{NH}_{3}$ vapors, the overall structure of the sample remains unchanged, exhibiting an entangled network but with slightly melted fibrils (Fig. 2B3). This shows that ammonia exposure does not affect the 3D organization of the collagen nanofibers. This is a very interesting alternative to crosslinking strategies usually used to stabilize collagen-based membranes. Unlike previous works, in this case no gelation process is induced: ammonia exposure allows for the membrane storage in a prefibrillated state. This can be attributed to the limited extent of fibrillation at the solid-vapor interface hampered by the low amount of water as diffusing medium. After hydration, large fibrils could be observed exhibiting the $67-\mathrm{nm}$ periodic pattern characteristic of collagen organization in living tissues (Fig. 2C3). Additional images can be found in the photo gallery of the Supplementary Materials to better highlight the nanostructure of collagen fibrils with the periodic pattern of 67 
nm (Fig. S7). These fibrils further organized into bundles exhibiting large width and polydispersity: from $200 \mathrm{~nm}$ to $2 \mu \mathrm{m}$, with a mean width of $560 \pm 290 \mathrm{~nm}$ over a population of 61 bundles (see histogram of bundle width distribution in Fig. 2D). This very nicely confirms the variations of the collagen network observed by SHG/pSHG and again proves the native state of collagen within ES membranes given that non-native collagen cannot form cross-striated fibrils. Very importantly, this ascertains the possibility to recover the self-assembling processes and hierarchical organization of collagen as observed in living tissues. This is the first time that such organization could be obtained after ES.

However, SHG and cryo-FE-SEM give a rather local view of the sample and we require at this stage to have the confirmation of collagen native state over the whole sample. To do so, we used synchrotron-radiation circular dichroism (SRCD) that gives the molecular signature of native collagen at the scale of the triple helix over the whole sample. Self-supported membranes were dissolved in $3 \mathrm{mM} \mathrm{HCl}$. A large negative band at $198 \mathrm{~nm}$ and a small positive band at $223 \mathrm{~nm}$ were observed at $20^{\circ} \mathrm{C}$ (Fig. $4 \mathrm{~A}$ solid line). This corresponds to the spectral signature of the collagen triple helix after extraction and before ES (Fig. 4A, dotted line). These bands are characteristic of the polyproline II (PPII) conformation of collagen triple helix. This CD spectrum strikingly deviates from previous studies that showed collagen denaturation, ${ }^{10,11}$ and confirms that collagen fully preserves its native state after ES. The melting temperature (Tm) of the soluble triple helix was measured by thermal denaturation with increasing temperature up to $74^{\circ} \mathrm{C}$ in steps of $3^{\circ} \mathrm{C}$. As expected, the intensity of both bands decreases with the temperature increase (Fig. 4B from dark blue to dark red). This is attributed to the increased fraction of unfolded structures upon thermal denaturation. Tm was determined by plotting the ellipticity at maxima (198 and $223 \mathrm{~nm}$ ) as a function of temperature using the sigmoidal fit in "Igor Pro" spectral analysis program. After 
$\mathrm{ES}$, the Tm was found to be around $31^{\circ} \mathrm{C}$ at $198 \mathrm{~nm}$ and $30.8^{\circ} \mathrm{C}$ at $223 \mathrm{~nm}$ (Fig. 4C), in agreement with previously reported values for native collagen. ${ }^{12}$ Above $31{ }^{\circ} \mathrm{C}$, the structure is dominated by unordered conformations, and collagen completely unfolds above $40^{\circ} \mathrm{C}$. To estimate the impact of the ES process, we compared the thermal denaturation of the electrospun triple helix with that of native collagen after extraction. Figure 4D shows that Tm of pure collagen is similar to the one of the ES membrane $\left(31.5^{\circ} \mathrm{C}\right.$ at $198 \mathrm{~nm}$ and $31.7^{\circ} \mathrm{C}$ at $\left.223 \mathrm{~nm}\right)$. This shows that the fraction of PPII at room temperature in both samples is the same and further confirms that collagen fully preserves its native state over the whole sample. 

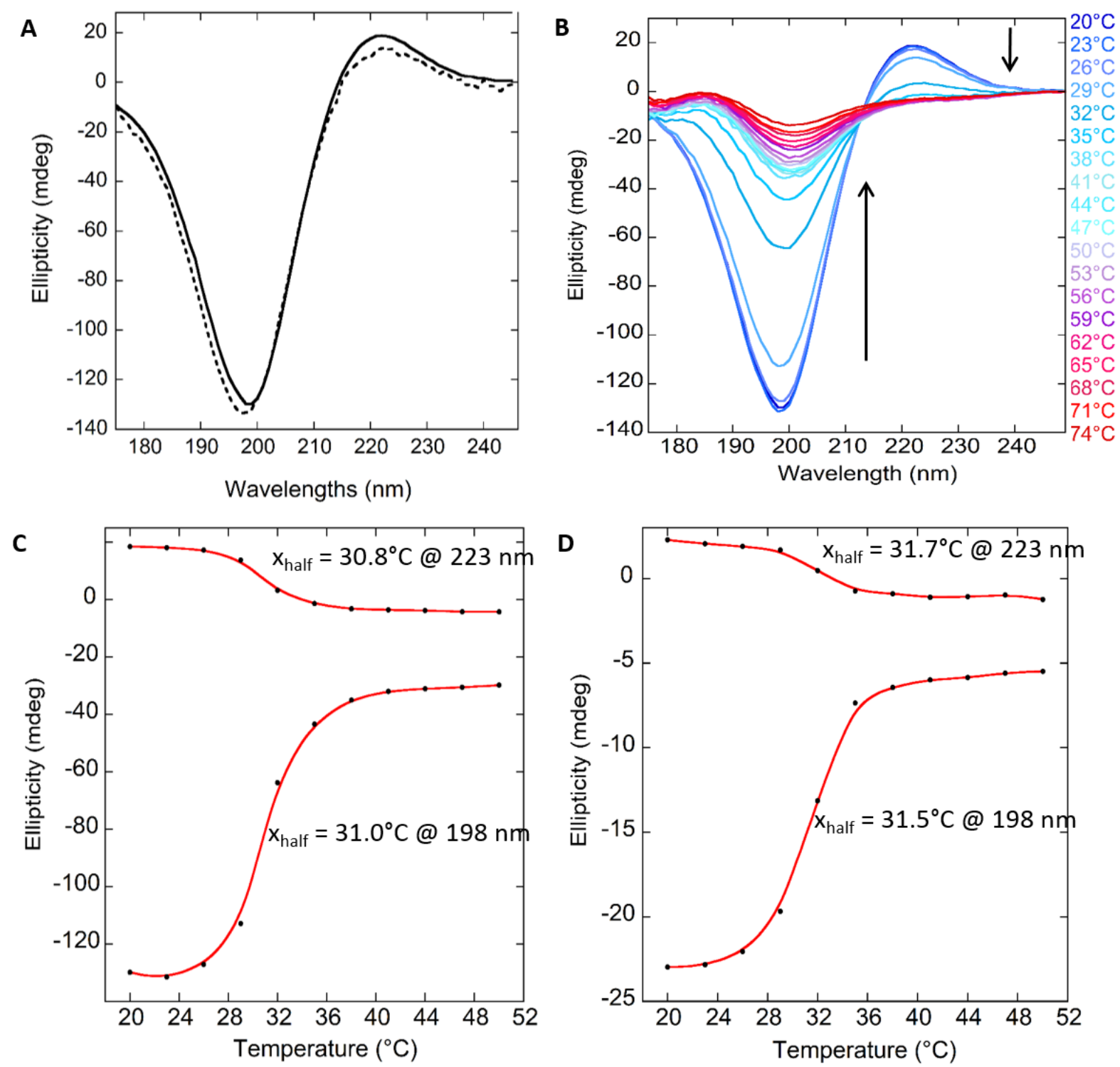

Figure 4. SRCD spectra of (A) collagen in $3 \mathrm{mM} \mathrm{HCl}$ before (dotted line) and after (solid line) ES, and of (B) thermal unfolding of ES collagen from 10 to $74{ }^{\circ} \mathrm{C}$ in steps of $3^{\circ} \mathrm{C}$. (C-D) Ellipticity as a function of temperature: Tm at 198 and $223 \mathrm{~nm}$ for ES and native collagen respectively.

\section{Biofabrication for a broad range of applications}

To assess the cytocompatibility and bioactivity of electrospun collagen nanofibers, cell attachment and spreading of normal human dermal fibroblast cells (NHDF) seeded onto the 
collagen matrix were studied. Immunostaining of actin and nuclei revealed the colonization of the scaffold, where the fibroblasts were spread all over the membrane and exhibited a spindle-like shape morphology with stress fibers clearly visible inside the cellular body (Fig. 5A, white arrows), showing that electrospun membrane are perfectly suitable for cell adhesion and spreading. No significant difference in terms of cell morphology and area could be observed when compared to control non-electrospun biomaterials, ie casted membranes obtained by solvent evaporation (Fig. 5B). Finally, the metabolic activity after one week was assessed revealing no significant difference between electrospun, casted and control support (Fig. 5C).
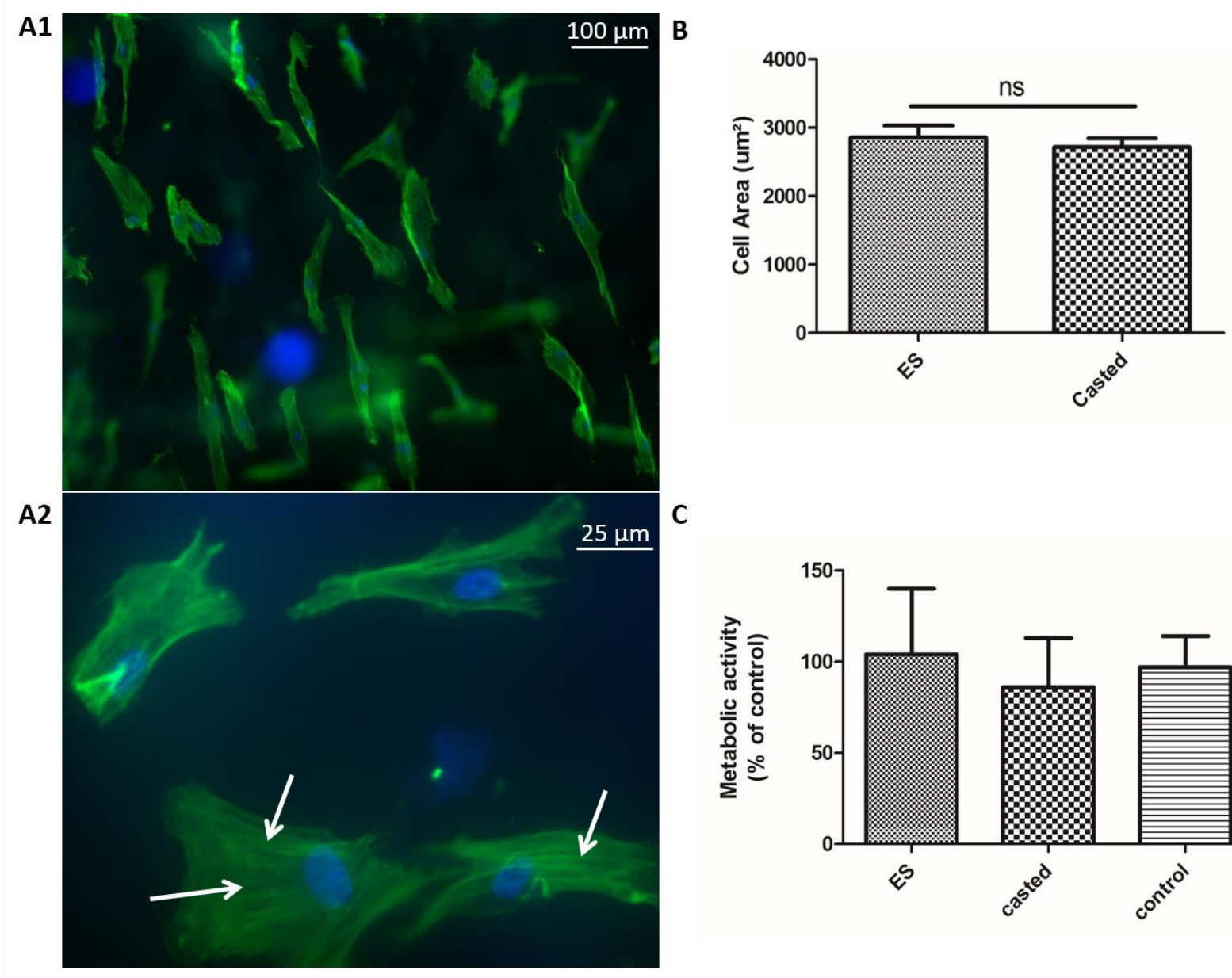

Figure 5. (A1-A2) Fluorescence microscopy images at different magnifications of NHDF cultured for $24 \mathrm{~h}$ on ES membranes. Actin (green: phalloidin) and nucleus (blue: DAPI) staining. White 
arrows show stress fibers. (B) Cell area of NHDFs seeded on electrospun and casted membranes $(\mathrm{n}=3)$. Statistical analysis performed using Mann-Whitney non parametric test $(*: \mathrm{p}<0.05)$. (C) Metabolic activity of NHDFs measured by Alamar blue assay after a one week culture on different supports (ES and casted collagen membrane, and control plastic support). The column represents Mean with SEM.

The processing of biopolymers with preserved native molecular conformation, hierarchical structure and interactions with cells is necessary to replicate biological microenvironments. This remains a huge challenge and a real need for the advancement of basic research in biology and biomedical research. With this challenge ahead, we first worked on the engineering of hybrid_or composite _ biomaterials obtained by mixing collagen with an inorganic phase. The composite approach is an efficient strategy to improve the functionality of bio-inspired matrices by synergistically combining biopolymers with colloids of different chemical nature. In this context, we added carbon nanotubes $(0.125 \%$ in weight $)$ to collagen before ES. Self- supported membranes could be obtained, in identical conditions to that of pure collagen, with a very good homogeneity in terms of carbon nanotube dispersion within the final biomaterials, as observed both macroscopically and under SEM (Fig. 6A, where carbon nanotubes are hardly distinguishable among collagen nanofibers). Alternatively, silica particles could successfully be electrospun and incorporated within a collagen-based self-supported membrane with good dispersion (Fig. 6B, white arrows). This shows that the ES of collagen is a robust process that allows adjusting the composition of the biopolymer solution for the electrospinning of inorganic colloids. In addition, and very importantly, the ES of collagen ensures a good dispersion of the two phases that would not be reached using a casting strategy based on solvent evaporation. Given the strength of the 
composite approach to design conductive, optical, magnetic devices, or tune their mechanical properties, ${ }^{43,44}$ collagen ES represents an important step for the engineering of collagen-based biomaterials.

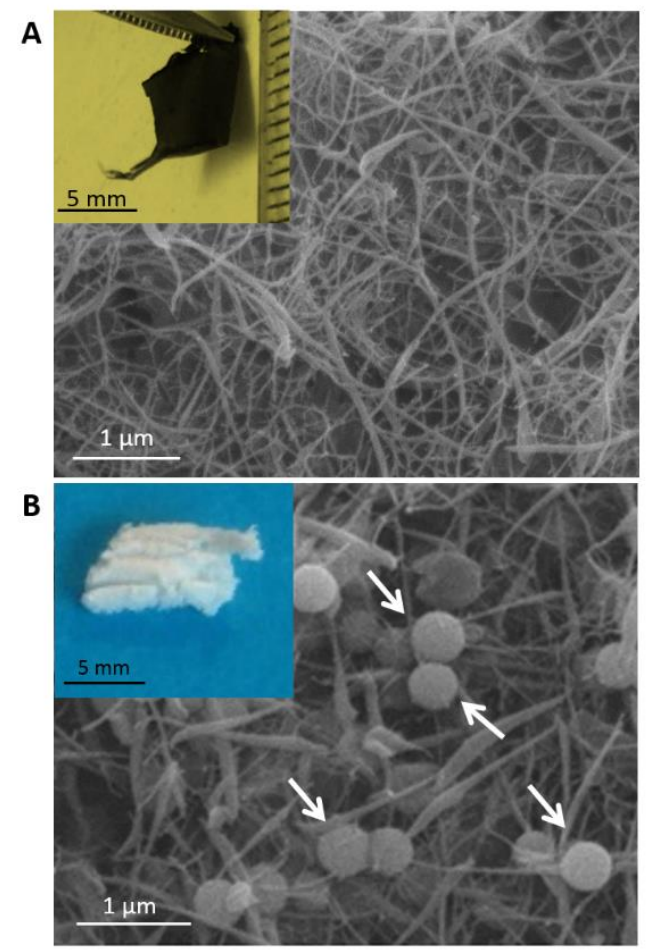

Figure 6. Photos (inserts) and SEM images of hybrid biomaterials combining collagen with (A) carbon nanotubes and (B) silica particles.

Another advantage in using ES is the possibility to easily control the thickness of the deposited membrane for the coating of a support of interest. This is particularly interesting in nano- and microtechnologies typically for the fabrication of microfluidic chips. Microfluidic chips are becoming crucial tools in biological and biomedical research by implementing molecular exchange and flux to in vitro models. It is thus of utmost importance to provide micro biochips with scaffolding that recapitulates the architecture and cell interactions of living systems. In this context, we used microfabricated frames to be incorporated into microfluidic devices as a support 
for collagen ES (Fig. 7A). These microframes have been shown to be highly suitable for cell culture and stem cell differentiation. ${ }^{45-48}$ In this case, collagen is electrospun directly onto the microframe as described above, and the microframe prefibrillated under ammonia vapor and hydrated in culture medium. In those conditions, SHG observations on top of the microframe showed the formation of submicron thick collagen layers with native hierarchical structure (Fig. 7B). This again shows the robustness of our protocol to produce ECM-like scaffolds made up of native type I collagen. This makes the ES of collagen a precious alternative to synthetic or nonnative biopolymers or to the use of hydrogels with variable thickness.

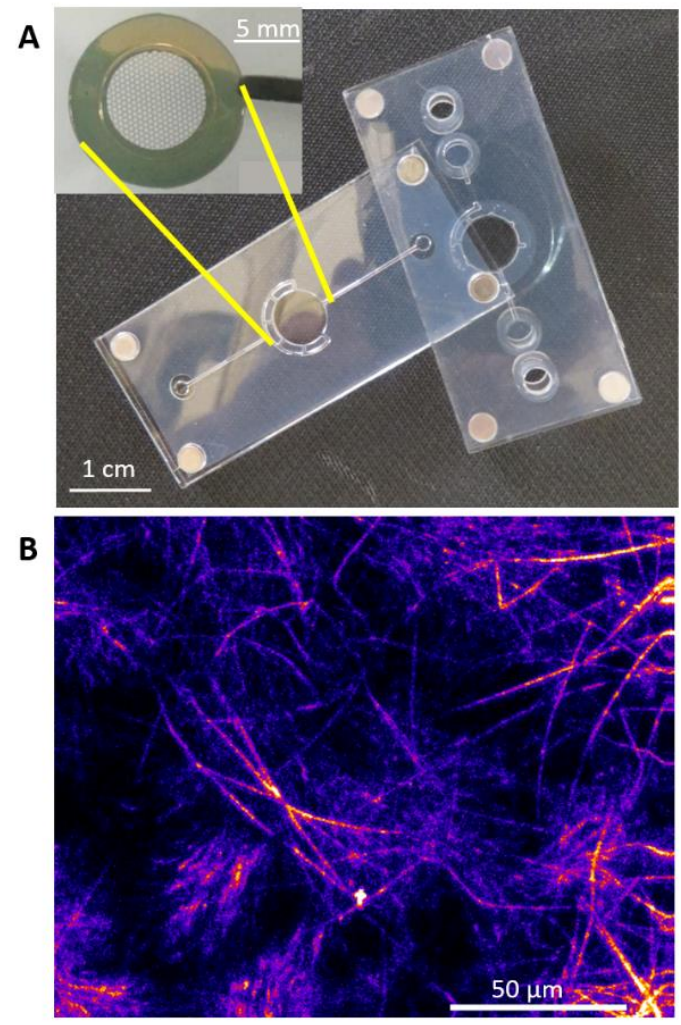

Figure 7. (A) Photo of the microfabricated frame and bio-chip before ES, and (B) SHG image of the coating of the microframe by collagen ES. 
CONCLUSION. Native type I collagen can be electrospun into a self-supported membrane in absence of cross-linker and polymer support. This is a prerequisite to improve in vitro models providing cells with the necessary biochemical and biophysical cues. We propose a methodology to thoroughly characterize collagen-based biomaterials using SHG, which is the most powerful optical probe for self-assembled type I collagen given its high specificity to triple helix packing within collagen fibrils, combined with cryo electron microscopy to preserve the hydrated state of the collagen membrane and SRCD as the gold biochemical standard to investigate the conformation of biomolecules. Altogether, this shows that collagen preserves its native conformation and ability to self-assemble into fibrils after ES. Dry electrospun collagen can be stabilized by pre-fibrillation in vapor state to avoid collapse upon hydration. This procedure allows to store dry membranes as pre-fibrillated scaffolds. This provides very easy to handle biomaterials of pure collagen, ready to use for biomedical applications including implantations, with preserved hierarchical structure and biological activity. Because of the final processing of ES materials into thin coatings and self-supported films, a broad range of applications can be targeted, including wound healing, cornea repair, heart patches and neural regeneration. We also show that this process can be applied to the coating of microchips for biological microfluidics with the possibility to adjust the composition of the biopolymer solution to be electrospun, notably towards hybrid materials. This opens many developments for the reconstruction of microenvironments with improved biological relevance for future advances in biological and biomedical research.

\section{ASSOCIATED CONTENT}

Supporting Information. Optimization of the solution conditions to improve collagen electrospinnability; Macroscopic structure of the fibrillary self-supported membrane; Passive 
degradation after one month in cell culture medium; Mechanical properties of the ES membrane; Photo gallery of the hydrated electrospun membrane by cryo-FE-SEM; Supplementary experimental section for passive degradation test and AFM nanoindentation and imaging.

\section{AUTHOR INFORMATION}

\section{Corresponding Author}

*carole.aime@ens.psl.eu

\section{Present Addresses}

$†$ Ecole Normale Supérieure, CNRS-ENS-SU UMR 8640, 24 rue Lhomond, Paris, 75005, France

\section{Author Contributions}

The manuscript was written through contributions of all authors. All authors have given approval to the final version of the manuscript.

\section{Funding Sources}

This work was partly supported by the Agence Nationale de la Recherche (contracts ANR-10INBS-04 France BioImaging and ANR-11-EQPX-0029 orphoscope2).

\section{Notes}

The authors declare no competing financial interest.

\section{ACKNOWLEDGMENT}

We thank Gervaise Mosser, Alexandre Bahezre for helpful discussions; Antoine Frayssinet for experimental help; Patrick Le Griel for his help in building the ES chamber; Ghislaine Frébourg, Virginie Bazin, Alexis Canette, Michael Trichet for cryo-FE-SEM observations performed at the 
Electron Microscopy Facility (EMF) of the Institut de Biologie Paris-Seine (FR3631, Sorbonne University, CNRS, Paris), Tristan Piolot from the microscopy platform at CIRB - Collège de France for his help with AFM experiments, Emmanuel Flahaut from the CIRIMAT-UMR CNRS 5085 for providing carbon nanotubes, and Margaux Schmeltz and Clothilde Raoux for their constant supports with SHG experiments. SRCD on DISCO beamline at the SOLEIL Synchrotron was performed under proposals 20180440 and 20170028. CA thanks Florence Babonneau for her constant support.

\section{ABBREVIATIONS}

CCR2, CC chemokine receptor 2; CCL2, CC chemokine ligand 2; CCR5, CC chemokine receptor 5; TLC, thin layer chromatography.

\section{REFERENCES}

1 Cui, W.; Zhou, Y.; Chang, J. Electrospun nanofibrous materials for tissue engineering and drug delivery. Sci Technol Adv Mater. 2010, 11, 014108.

2 Kitsara, M.; Agbulut, O.; Kontziampasis, D.; Chen, Y.; Menasché, P. Fibers for hearts: A critical review on electrospinning for cardiac tissue engineering. Acta Biomater. 2017, 48, 20-40.

3 Dror, Y.; Ziv, T.; Makarov, V.; Admon, A.; Zussman, E. Nanofibers made of globular proteins. Biomacromolecules 2008, 9, 2749-2754.

4 Regev, O.; Vandebril, S.; Zussman, E.; Clasen, C. The role of interfacial viscoelasticity in the stabilization of an electrospun jet. Polymer 2010, 51, 2611-2620.

5 Matthews, J. A.; Wnek, G. E.; Simpson, D. G.; Bowlin, G. L. Electrospinning of collagen nanofibers. Biomacromolecules 2002, 3, 232-238. 
Electrospun protein fibers as matrices for tissue engineering. Biomaterials 2005, 26, 5999-6008.

7 Kwon, I. K.; Matsuda, T. Co-electrospun nanofiber fabrics of poly(L-lactide-co-epsiloncaprolactone) with type I collagen or heparin. Biomacromolecules 2005, 6, 2096-2105.

8 Zhang, Y. Z.; Venugopal, J.; Huang, Z. M.; Lim, C. T.; Ramakrishna, S. Characterization of the surface biocompatibility of the electrospun PCL-collagen nanofibers using fibroblasts. Biomacromolecules 2005, 6, 2583-2589.

9 Chen, Z.; Mo, X.; Qing, F. Electrospinning of collagen-chitosan complex. Mater. Lett. 2007, 61, 3490-3494.

10 Yang, L.; Fitié, C. F. C.; van der Werf, K. O.; Bennink, M. L.; Dijkstra, P. J.; Feijen, J. Mechanical properties of single electrospun collagen type I fibers. Biomaterials, 2008, 29, 955962.

11 Zeugolis, D. I.; Khew, S. T.; Yew, E. S.; Ekaputra, A. K.; Tong, Y. W.; Yung, L. Y;. Hutmacher, D. W.; Sheppard, C.; Raghunath, M. Electro-spinning of pure collagen nano-fibres just an expensive way to make gelatin? Biomaterials 2008, 29, 2293-2305.

12 Bürck, J.; Heissler, S.; Geckle, U.; Ardakani, M. F.; Schneider, R.; Ulrich, A. S.; Kazanci, M. Resemblance of electrospun collagen nanofibers to their native structure. Langmuir 2013, 29, 1562-1572.

13 Dong, B.; Arnoult, O.; Smith, M. E.; Wnek, G. E. Electrospinning of collagen nanofiber scaffolds from benign solvents. Macromol. Rapid Commun. 2009, 30, 539-542. 
14 Elamparithi, A.; Punnoose, A. M.; Kuruvilla, S. Electrospun type 1 collagen matrices preserving native ultrastructure using benign binary solvent for cardiac tissue engineering. Artif. Cells Nanomed. Biotechnol. 2016, 44, 1318-1325.

15 Rho, K. S.; Jeong, L.; Lee, G.; Seo, B. M.; Park, Y. J.; Hong, S. D.; Roh, S.; Cho, J. J.; Park, W. H.; Min, B. M. Electrospinning of collagen nanofibers: effects on the behavior of normal human keratinocytes and early-stage wound healing. Biomaterials 2006, 27, 1452-1461.

16 Barnes, C. P.; Pemble, C. W.; Brand, D. D.; Simpson, D. G.; Bowlin, G. L. Cross-linking electrospun type II collagen tissue engineering scaffolds with carbodiimide in ethanol. Tissue Eng. 2007, 13, 1593-1605.

17 Shih, Y. R.; Chen, C. N.; Tsai, S. W.; Wang, Y. J.; Lee, O. K. Growth of mesenchymal stem cells on electrospun type I collagen nanofibers. Stem Cells 2006, 24, 2391-2397.

18 Buttafoco, L.; Kolkman, N. G.; Engbers-Buijtenhuijs, P.; Poot, A. A.; Dijkstra, P. J.; Vermes, I.; Feijen, J. Electrospinning of collagen and elastin for tissue engineering applications. Biomaterials 2006, 27, 724-734.

19 Huang, G. P.; Shanmugasundaram, S.; Masih, P.; Pandya, D.; Amara, S.; Collins, G.; Arinzeh, T. L. An investigation of common crosslinking agents on the stability of electrospun collagen scaffolds. J Biomed Mater Res A. 2015, 103, 762-771.

20 Tillman, B. W.; Yazdani, S. K.; Lee, S. J.; Geary, R. L.; Atala, A.; Yoo, J. J. The in vivo stability of electrospun polycaprolactone-collagen scaffolds in vascular reconstruction.

Biomaterials 2009, 30, 583-588. 
21 Dippold, D.; Cai, A.; Hardt, M.; Boccaccini, A. R.; Horch, R.; Beier, J. P.; Schubert, D. W. Novel approach towards aligned PCL-Collagen nanofibrous constructs from a benign solvent system. Mater. Sci. Eng. C Mater. Biol. Appl. 2017, 72, 278-283.

22 Huang, L.; Nagapudi, K.; Apkarian, R. P.; Chaikof, E. L. Engineered collagen-PEO nanofibers and fabrics. J. Biomater. Sci. Polymer Ed. 2001, 12, 979-993.

23 Huang, L.; Apkarian, R. P.; Chaikof, E. L. High-resolution analysis of engineered type I collagen nanofibers by electron microscopy. Scanning 2001, 23, 372-375.

24 Wakuda, Y.; Nishimoto, S.; Suye, S. I.; Fujita, S. Native collagen hydrogel nanofibres with anisotropic structure using core-shell electrospinning. Sci. Rep. 2018, 8, 6248.

25 Schiffman, J. D.; Schauer, C. L. A Review: Electrospinning of Biopolymer Nanofibers and their Applications Polym. Rev. 2008, 48, 317-352.

26 Li, M.; Mondrinos, M. J.; Gandhi, M. R.; Ko, F. K.; Weiss, A. S.; Lelkes, P. I. Electrospun protein fibers as matrices for tissue engineering. Biomaterials, 2005, 26, 5999-6008.

27 Kidoaki, S.; Kwon, I. K.; Matsuda, T. Mesoscopic spatial designs of nano- and microfiber meshes for tissue-engineering matrix and scaffold based on newly devised multilayering and mixing electrospinning techniques. Biomaterials 2005, 26, 37-46.

28 Gobeaux, F.; Mosser, G.; Anglo, A.; Panine, P.; Davidson, P.; Giraud-Guille, M.-M.; Belamie, E. Fibrillogenesis in dense collagen solutions: a physicochemical study. J. Mol. Biol. 2008, 376, 1509-1522. 
29 Giraud-Guille, M.-M.; Besseau, L.; Herbage, D.; Gounon, P. Optimization of Collagen Liquid-Crystalline Assemblies - Influence of Sonic Fragmentation. J. Struct. Biol. 1994, 113, 99106.

30 Bergman, I.; Loxley, R. Two Improved and Simplified Methods for the Spectrophotometric Determination of Hydroxyproline. Anal. Chem. 1963, 35, 1961-1965.

31 Giuliani, A.; Jamme, F.; Rouam, V.; Wien, F.; Giorgetta, J.-L.; Lagarde, B.; Chubar, O.; Bac, S.; Yao, I.; Rey, S.; Herbeaux, C.; Marlats, J.-L.; Zerbib, D.; Polack, F.; Réfrégiers, M. DISCO: a low-energy multipurpose beamline at synchrotron SOLEIL. J. Synchrotron Radiat. 2009, 16, 835-841.

32 Wien, F; Paternostre, M.; Gobeaux, F.; Artzner, F.; Refregiers, M. J. Phys.: Conf. Ser. 2013, 425, 122014.

33 Lees, J. G.; Smith, B. R.; Wien, F.; Miles, A. J.; Wallace, B. A. CDtool-an integrated software package for circular dichroism spectroscopic data processing, analysis, and archiving. Anal. Biochem. 2004, 332, 285-289.

34 Teulon, C.; Tidu, A.; Portier, F.; Mosser, G.; Schanne-Klein, M.-C. Probing the 3D structure of cornea-like collagen liquid crystals with polarization-resolved SHG microscopy. Opt. Express 2016, 24, 16084-16098.

35 Cui, W.; Li, X.; Zhou, S.; Weng, J. Investigation on process parameters of electrospinning system through orthogonal experimental design. J. Appl. Polym. Sci. 2007, 103, 3105-3112. 
36 Giraud-Guille, M.-M.; Besseau, L.; Martin, R. Liquid crystalline assemblies of collagen in bone and in vitro systems. J. Biomech. 2003, 36, 1571-1579.

37 Aimé, C.; Mosser, G.; Pembouong, G.; Bouteiller, L. Coradin, T. Nanoscale 2012, 4, 7127-7134.

38 Wang, X.; Yan, Y.; Xiong, Z.; Lin, F.; Wu, R.; Zhang, R.; Lu, Q. Preparation and evaluation of ammonia-treated collagen/chitosan matrices for liver tissue engineering. J. Biomed. Mater. Res. B Appl. Biomater. 2005, 75, 91-98.

39 Bancelin, S.; Aimé, C.; Coradin, T.; Schanne-Klein, M.-C. In situ three-dimensional monitoring of collagen fibrillogenesis using SHG microscopy. Biomed. Opt. Express 2012, 6, 1446-1454.

40 Bancelin, S.; Aimé, C.; Gusachenko, I.; Kowalczuk, L.; Latour, G.; Coradin, T.; Schanne-Klein, M.-C. Determination of collagen fibril size via absolute measurements of second-harmonic generation signals. Nat. Commun. 2014, 5, 4920.

41 Chen, X.; Nadiarynkh, O.; Plotnikov, S.; Campagnola, P. J. Second harmonic generation microscopy for quantitative analysis of collagen fibrillar structure. Nat. Protoc. 2012, 7, 654669.

42 Portier, F.; Teulon, C.; Nowacka-Perrin, A.; Genneau, F.; Schanne-Klein, M.-C., Mosser, G. Stabilization of Collagen Fibrils by Gelatin Addition: A Study of Collagen/Gelatin Dense Phases. Langmuir 2017, 33, 12916-12925. 
43 Aimé, C.; Coradin, T. Nanocomposites from biopolymer hydrogels: Blueprints for white biotechnology and green materials chemistry. J. Polym. Sci. Part B: Polym. Phys. 2012, 50, 669680.

44 Bionanocomposites: Integrating Biological Processes for Bioinspired Nanotechnologies. C. Aimé, T. Coradin ed. Wiley 2017.

45 Tang, Y.; Liu, L.; Li, J.; Yu, L.; Severino, F. P. U.; Wang, L.; Shi, J.; Tu, X.; Torre, V.; Chen, Y. Effective motor neuron differentiation of hiPSCs on a patch made of crosslinked monolayer gelatin nanofibers. J. Mater. Chem. B 2016, 4, 3305-3312.

46 Tang, Y.; Liu, L.; Li, J.; Yu, L.; Wang, L.; Shi, J.; Chen, Y. Induction and differentiation of human induced pluripotent stem cells into functional cardiomyocytes on a compartmented monolayer of gelatin nanofibers. Nanoscale 2016, 8, 14530-14540.

47 Liu, L.; Kamei, K. I.; Yoshioka, M.; Nakajima, M.; Li, J.; Fujimoto, N.; Terada, S.; Tokunaga, Y.; Koyama, Y.; Sato, H.; Hasegawa, K.; Nakatsuji, N.; Chen, Y. Nano-on-micro fibrous extracellular matrices for scalable expansion of human ES/iPS cells. Biomaterials 2017, $124,47-54$.

48 Tang, Y.; Severino, F. P. U.; Isepponn, F.; Torre, V.; Chen, Y. Patch method for culture of primary hippocampal neurons Microelectron. Eng. 2017, 175, 61-66.

SYNOPSIS (Word Style "SN_Synopsis_TOC"). 


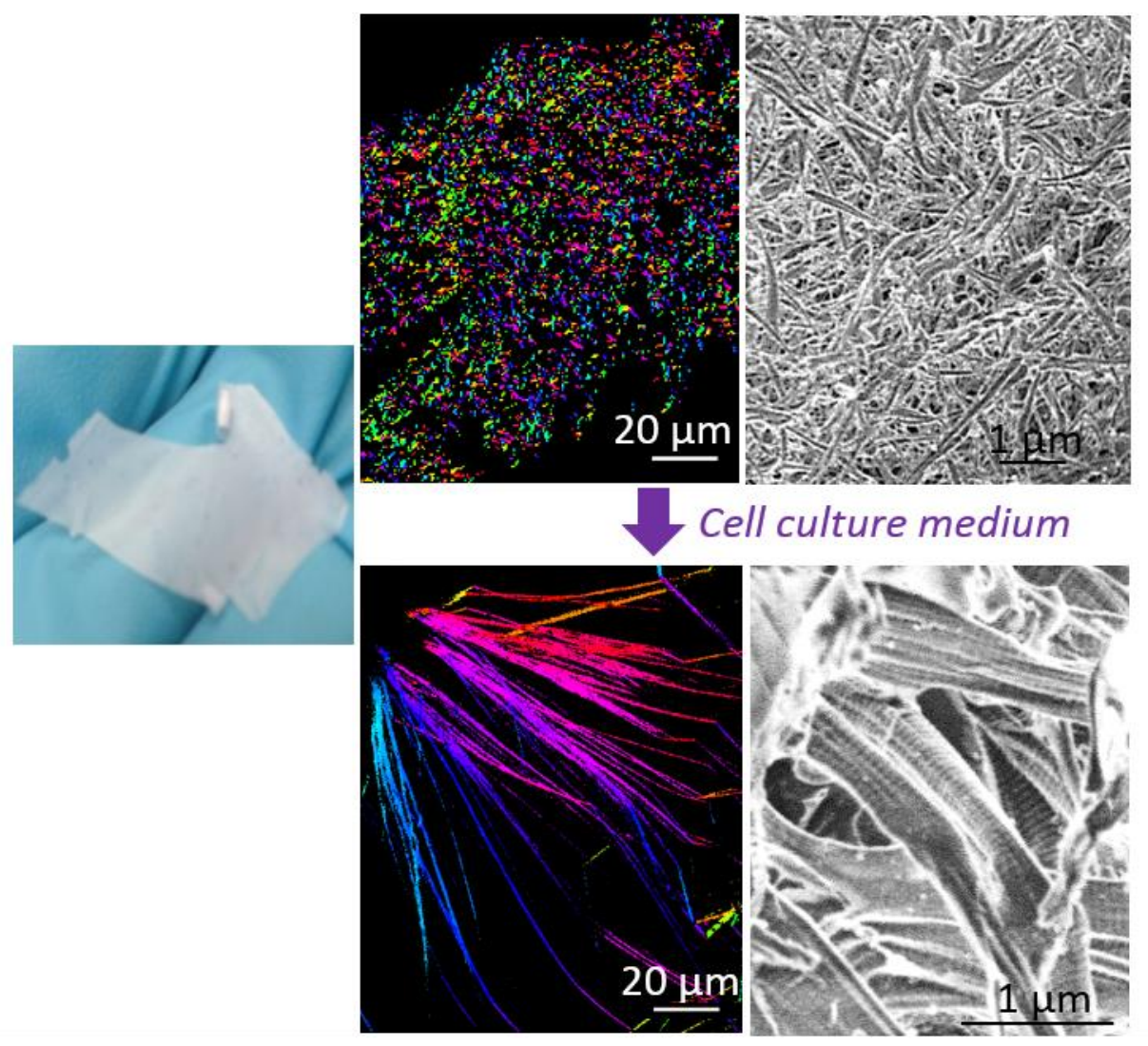

